\title{
Multi hop mechanism used in energy saving for multimedia file transfer in school education system
}

\author{
Zhe Zhou ${ }^{1}$, Jianying $\mathrm{Li}^{2}$ and Shuang Chen ${ }^{3}$ \\ ${ }^{1,2,3}$ Colloge of Education, Hebei Normal University of Science \& Technology, \\ Qinhuangdao, Hebei P.R. China,066004 \\ ${ }^{1}$ zhouzhets_2009@126.com, ${ }^{2} 154157362 @ q q . c o m, 3$ ss3399@126.com
}

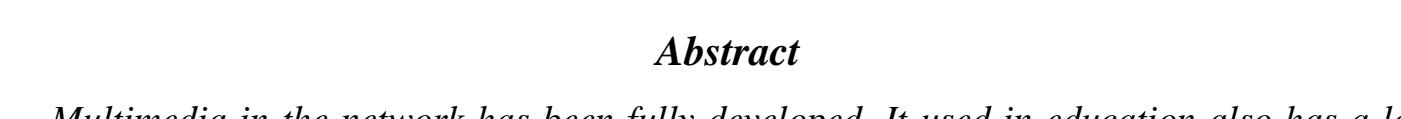

Multimedia in the network has been fully developed. In wsed in eduration also has a long history and resources in education network system become vern rich. Now, number of multimedia files in school server is still increasing now How termind computers in each place in school get multimedia files at fast speed with low entrgy consumption is a problem worthy to solve. In order to solve the problem, new method of dynamic algorithm based on PSO (Particle Swarm Optimization) and ACQ (ant colony optimization) method has been developed. This method is based on peer to peer mechanism and can be used in school with different network speed area. In the algorithm, the whole network is firstly divided into several subsystems. Each subsystem would be composed of computers with different network speed. One terminal computer would get multimedia files through computers with high network speed. This method can get high speed in fetching multimedia files and decrease energy consumption. The Gethod woula be firstly used in the education system then popularize into other systems.

Keywords: education network Syytem; streaming multimedia; multi hop; PSO; ACO

\section{Introduction}

Multimedia has greatlyychanged education system. Its vivid pictures and videos make the students concentrate on learning and improve efficiency and help teachers give students much more clear expression. We can find its application in nearly all the subjects where the network exists, including art teaching [1-2], film teaching [3], engineering education [4-7], even mathematics [8-9], and so on. Research of multimedia used in education network system has been greatly developed. It mainly focuses on some fields, including education methods [1011], teaching systems [12-13] and teaching tools [14-15]. Developing trend of education is also researched [16].

For the reason of various kinds of multimedia and large number of multimedia files, each terminal computer can't store all the learning resources. These resources are often stored in the server and distributed by proxy. When one terminal computer sends a request for some multimedia files, the proxy would fetch them from server and deliver them to the terminal computer. In order to keep watching or learning fluency, the fetching efficiency must be improved. In the paper, we would mainly focus on the file fetching mechanism and develop a new algorithm for the education system.

The system is shown in figure 1. We would like to define some items firstly. All the personal computers would be defined as terminal computers. In the system, peer to peer 
mechanism would be used to deliver or distribute the file data. Multimedia files as resources would be stored in server and all the terminal computers should request the resource according to the proxy. Streaming media technology would be used to transfer the data. The proxy fetches the data and sends them to terminal computers and terminal computers can also fetch data from other terminal computers. This mechanism would decrease the demand of bandwidth and improve the efficiency of terminal computers fetching data. If we make proper path, the data transmission would get the aim of energy saving. So, algorithm for terminal computers get multimedia files with high efficiency is very important.

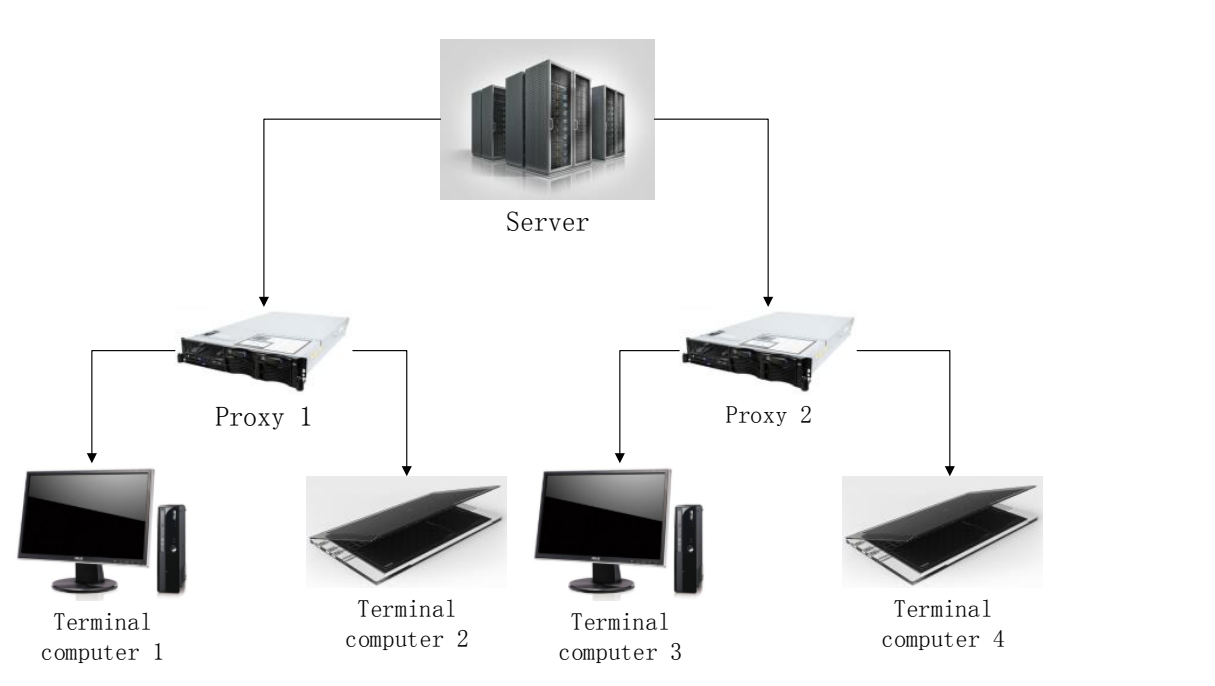

Figure 1. Proxy based Streaming Multimedia System

In the internet system, various algorithms in keeping multimedia watching and playing smoothly have been developed But the education system, especially in school education network systern, relative algorithm has not so many studies due to its own characteristics. For example, curriculum distribution in the terms is almost the same at the same time each year. Terminal computers may concurrently request for same resources while in internet, the request is random or uniform at same period in each year. So, algorithms are also different. However, the two systems have something in common in using special mechanism, for example, steaming media mechanism.

In the paper, we still follow the mechanism of streaming multimedia. It would firstly divide file-into segments and caching in proxy and then transfer data transmission in a special path. Segments dividing would use common method [17] and caching methods also have relativestudies [18]. Then, data transfer mechanism to keep playing or fetching smoothly is the most important part in the paper.

The main contribution of this paper is to develop a new data transfer mechanism. It is based on streaming media and to keep the data transfer fluency. In the method, whole education network is divided into several subsystems. In each subsystem, terminal computers with different network speeds are composed. Subsystem dividing method is based on PSO. After the dividing process is finished, multi hop mechanism based on ACO would be used in the terminal computers data fetching from the proxy and other computers. The method can improve the efficiency of data transferring and decrease the bandwidth consumption. The remainder of the paper is organized as the following: Basic algorithms are introduced in 
Section 2. Data transfer method is derived in Section 3. The example is given in section 4 and the conclusion is in Section 5.

\section{Algorithm Introduction}

\subsection{PSO Method}

PSO can be used to obtain the best results with the cooperation and shared information among each particle. It is simple to deal with the problems without adjusting many parameters.

Hypothesis:

(1) Particle location: $X_{i}=\left(x_{1}, x_{2}, \ldots, x_{N}\right)$;

(2) Velocity: $V_{i}=\left(v_{1}, v_{2}, \ldots, v_{N}\right)$;

(3) Best location of node knows: pbest

(4) Best place of each node found by the whole swarm: gbest

(5) PSO obtains the initialization and the best answer.

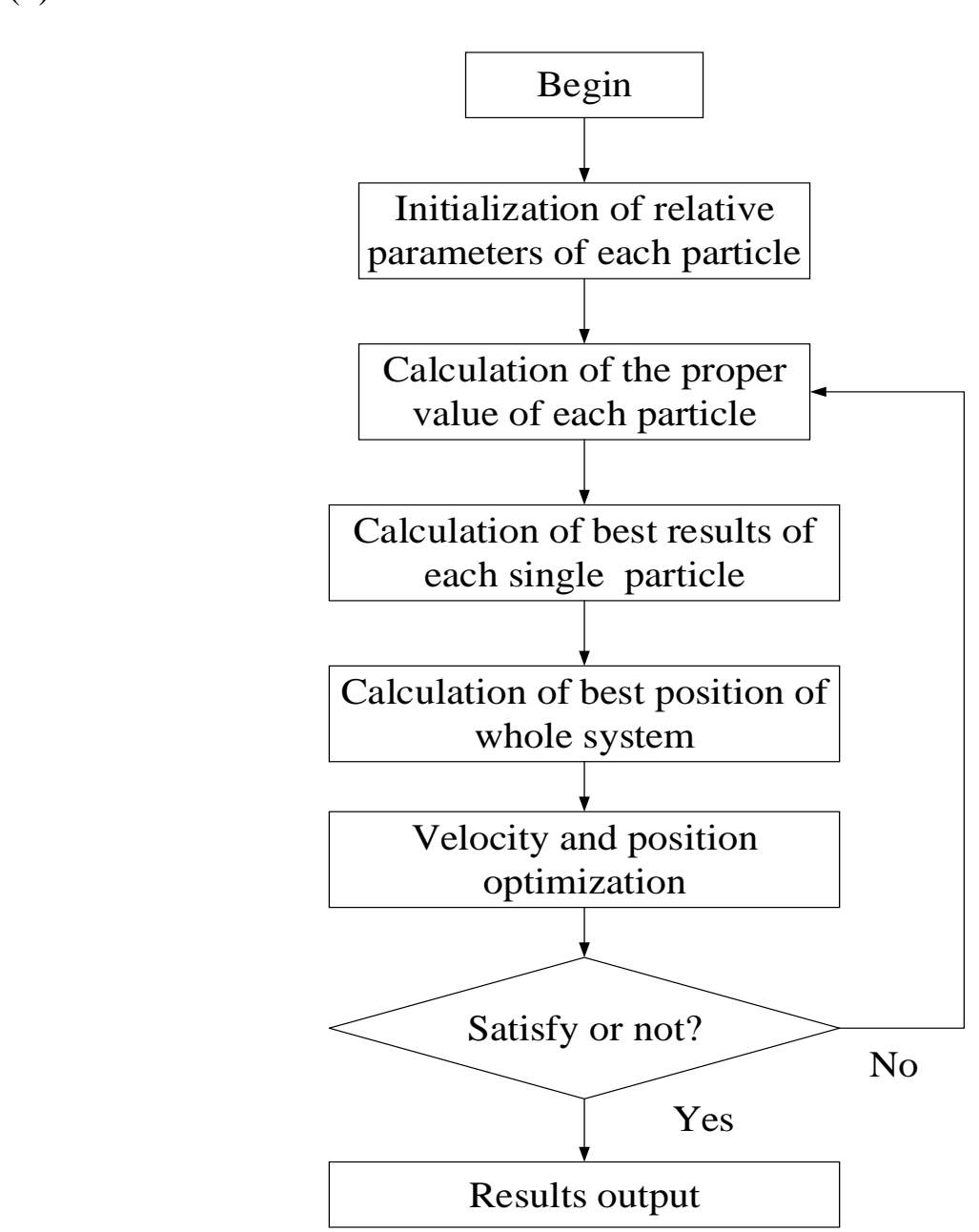

Figure 2. Generous Flowchart of PSO Method 
In each iterating step, value of pbest and gbest would be updated by the particle.

$$
\begin{gathered}
V_{i}=V_{i}+c_{1} \times \text { rand } \mathrm{O} \times\left(\text { pbest }_{i}-x_{i}\right)+c_{2} \times \text { rand } \mathrm{O} \times\left(\text { gbest }_{i}-x_{i}\right) \\
x_{i}=x_{i}+V_{i}
\end{gathered}
$$

Where, $i=1,2,3, \ldots, M, M$ is the total number of the swarm.

Equation (1) and (2) are the basic description of PSO, and they can be adjusted with the expression as the following:

$V_{i}=\omega \times V_{i}+c_{1} \times$ rand ()$\times\left(\right.$ pbest $\left._{i}-x_{i}\right)+c_{2} \times$ rand ()$\times\left(\right.$ gbest $\left._{i}-x_{i}\right)$

Where, $\omega$ is a factor influencing the balance between global search and local search 1 it can be a positive constant or even a positive linear or nonlinear function of time [19]. PSO has been used in various conditions. The general flowchart of PSO as Figure 2 shows.

\subsection{ACO}

The ACO algorithm is mainly used in solving optimization problems [20-22]. The optimization process can be seen as an ant looking for food. The algorithm is widely used in path searching in WSNs. A simplified and basic chart of ACO can be expressed in the Figure 3. For stability, ACO has been also developed [23].

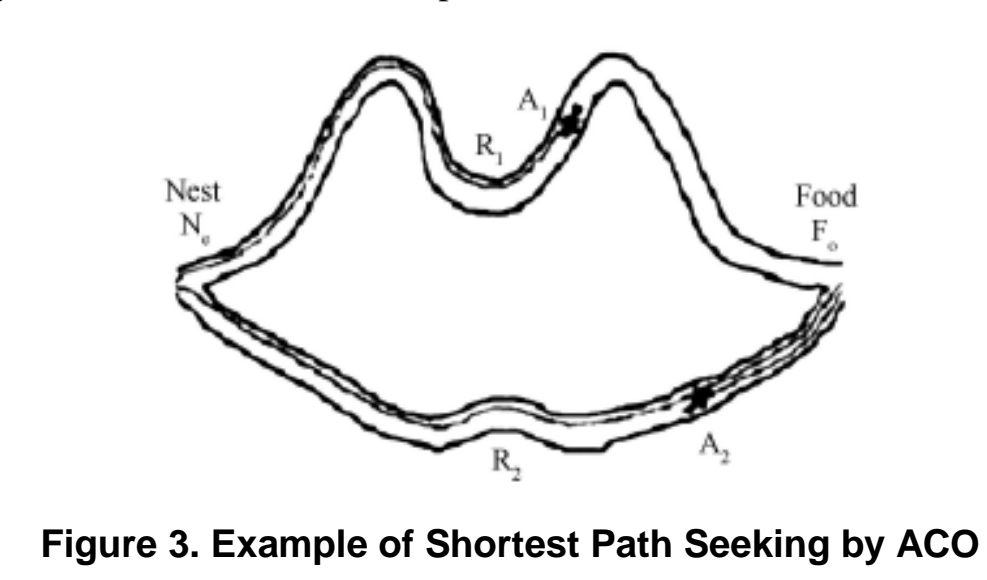

Firstly, we should give some introduction. There is a nest $N_{e}$ and a food sources $F_{0}$ in the Figure 3. Tw ants $A_{1}, A_{2}$ are supposed to find food along two roads $R_{1}, R_{2}$. Ants $A_{1}$ and $A_{2}$ have the same moving speed. The process can be described as follows:

(1) No ants in $N_{e}$ knows the exact position of the food source $F_{0}$. They would randomly select the path. We suppose that roads of $R_{1}$ and $R_{2}$ are selected by ant $A_{1}$ and $A_{2}$ respectively.

(2) When ant $A_{1}$ and ant $A_{2}$ moves along road $R_{1}$ and $R_{2}$ respectively, both of them would leave the same dose of pheromone at one time. Mark $T_{R 1}$ and $T_{R 2}$ as the pheromone density left by ant $A_{1}$ and ant $A_{2}$.

(3) Due to the shorter road length of $R_{2}$ than $R_{1}$, ant $A_{2}$ would get to food source $F_{0}$ earlier than ant $A_{1}$. While ant $A_{2}$ returns, it would still select $\operatorname{road}_{A_{2}}$ to food resource $F_{0}$ because of the higher density of pheromone on $\operatorname{road} R_{2}$ than that at $R_{1}$. It means that value of $T_{R 1}$ is 
smaller than that of $T_{R 2}$. When it returns, it leaves the same dose of pheromone and road $R_{2}$ has been strengthened. That means that $R_{2}$ is shorter path.

(4) If ant $A_{1}$ arriving at $F_{0}$, it can find that density of pheromone on road $R_{2}$ is double times than that at $R_{1}$. Then ant $A_{1}$ would select road $R_{2}$ to return.

Finally, both ant $A_{1}$ and ant $A_{2}$ return along the shorter $\operatorname{road}_{R_{2}}$. Figure 4 shows the general flowchart of ACO.

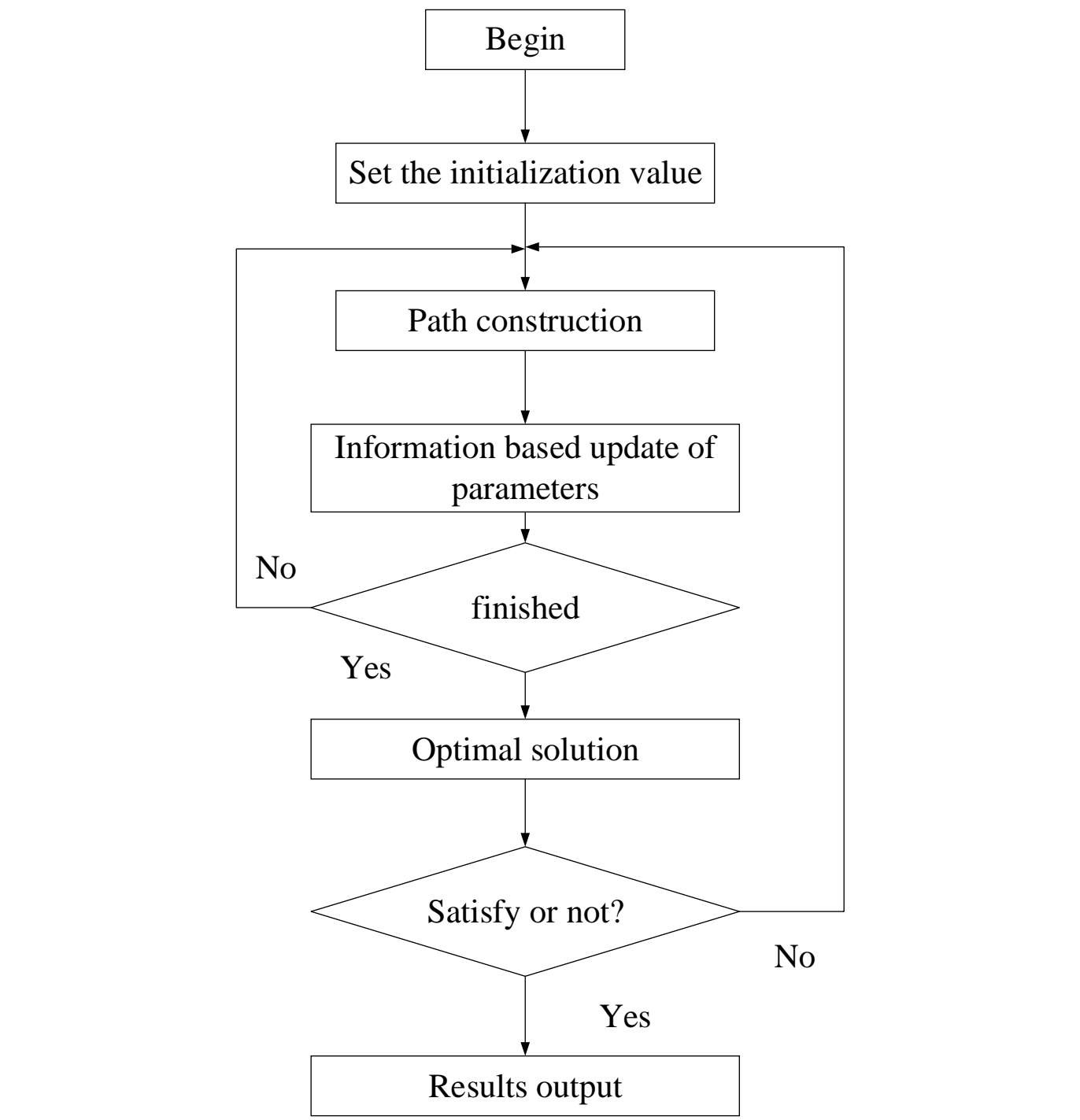

Figure 4. Flowchart of ACO

\section{Multi Hop Data Transfer Algorithm}

Multi-hop data transfer algorithm is aimed at high speed and low energy consumption in data transfer. 


\subsection{Main Idea of Multi Hop Data Transfer Algorithm}

It is very important to keep data transfer at a proper speed to provide the fluency of file playing. Here a new algorithm of data transfer has been proposed to increase the data transfer speed and decrease the energy consumption. The algorithm includes some main steps as following:

Step 1: It would be optimized to determine proper computers number and distribution of computers with different network speed. In the school education system, different network speed would be set as three levels. Computers in classrooms have the fastest speed, computers in offices have the middle speed and computers in dormitories have the slowest speed. Computers with low speed can get data from proxy and other computers with higher speed. In the latter mode, computers with higher network speed would cache the data required and low speed computers get the data from higher network speed computers. The aim of his step is to help to divide the whole system into subsystem.

Step 2: Particle swarm optimization would be used to diyide the network into more than one subsystem and ensure each subsystem gets computers with different network speed;

Step 3: In one subsystem, computers can get data from any omputers with higher network speed or directly from the proxy. ACO would beased to fix the fastest data transfer path.

The flow chart would be shown in Figure 5.

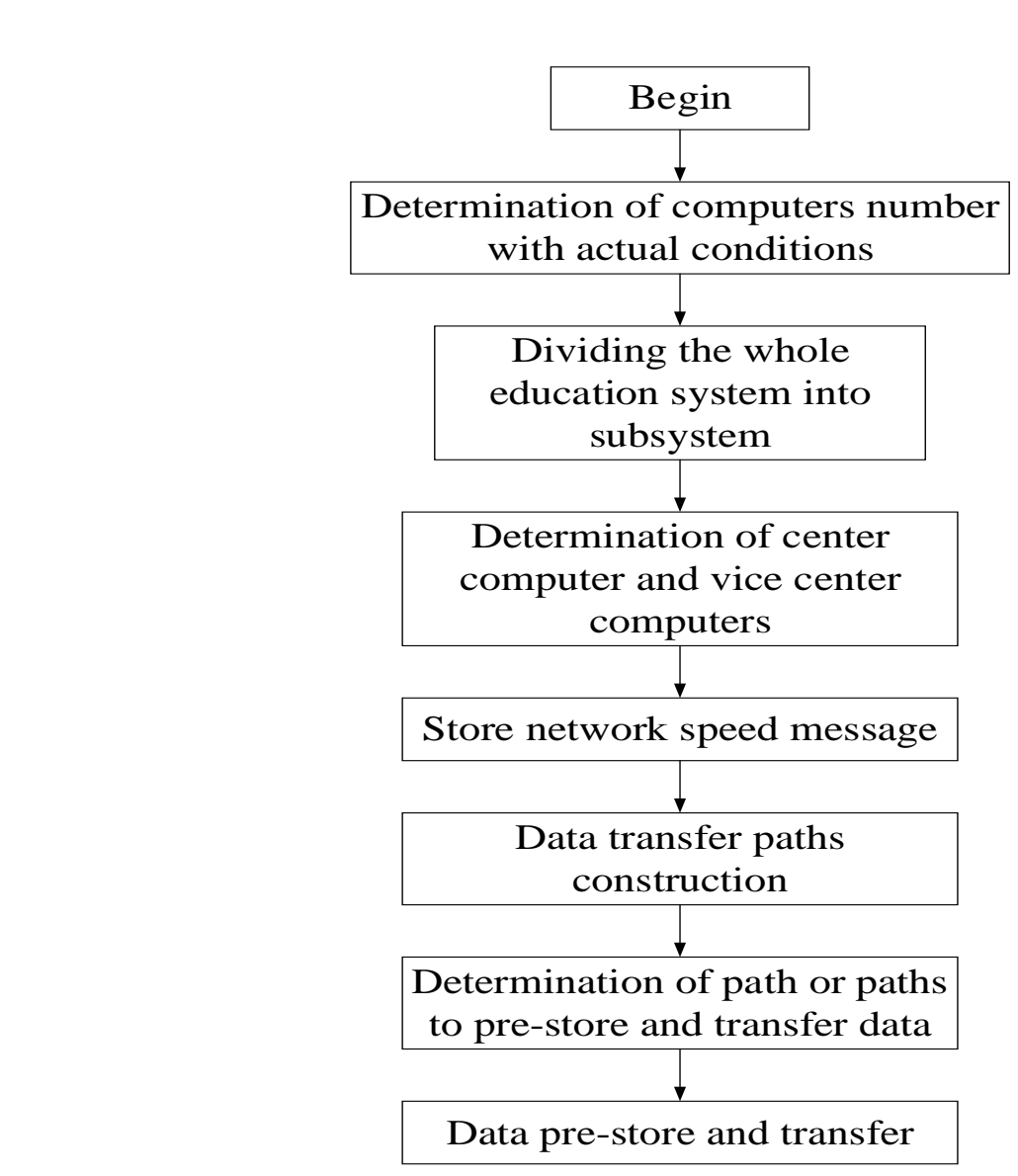

Figure 5. General Flow Chart of Data Transfer Algorithm 


\subsection{The Detail Steps of Algorithm}

\section{Step 1: Determination of the nodes number}

In wireless sensor network, this question has been widely studied [24-25]. But the education system is different from the wireless sensor network. The division in education system is mainly for higher download speed and low energy consumption. In the division, network speed is the main factor. In order to provide each computer get enough data transfer speed, we use circular disk model to divide the whole education system into several subsystem with proper number of computers. As shown in Figure 6. A computer with the highest network speed would be in the center of subsystem and with the name of center computer. The center computer is contact with other computers with cables and center computers would also contact with proxy and server with cables. Due to the connection of each computer to center computer or other computers with cable, all computers in the whole education system would be covered.

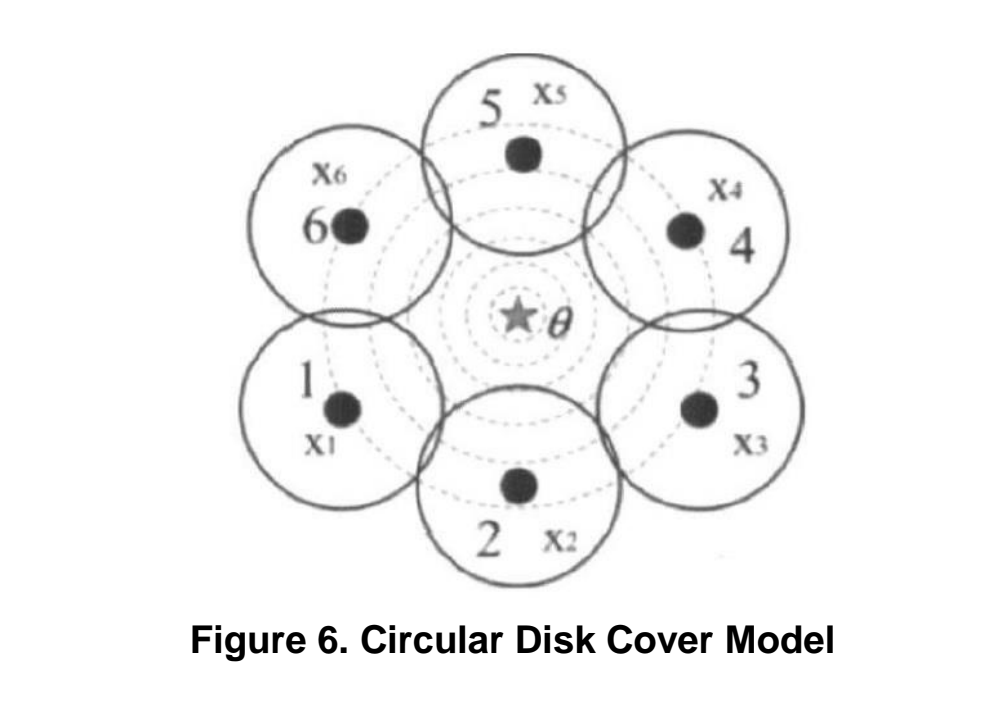

Step 2: Division of the edueation system into subsystem

(1) Firstly, an assumption would be given: there are $\mathrm{N}$ computers in the network, and all the computers would be split into $\mathrm{M}$ subsystem. There would be $N / M$ computers in each subsystem. Dratv a splitter to divide the whole system into two domains with same number of access to proxy. The split line can be described as the following:

$$
U=(x, y, \theta)
$$

Where, $(x, y)$ is location of nodes at the splitter line, $\theta$ is the angular formed by the splitter and $x$-axle.

Define function of fitness as follows:

$$
\text { fitness }=\left(c_{1}-f_{1} N\right)^{2}+\left(c_{2}-f_{2} \mathrm{~N}\right)^{2}
$$

In the equation, $c_{i}(i=1,2)$ represents nodes number in domain $i \cdot f_{i}$ can be determined by the equation:

$$
f_{i}=\frac{M_{i}}{M} \quad i=(1,2)
$$

Where, $M_{i}$ represents expectation of computers number in domain $i$.

Then, the first division has been finished. 
The algorithm of subsystem division has some steps as the following:

(1) All computers in the network send one special data segment to server. Server would get network condition of each computer. With the network condition, server would classify the level of each computer and send the information to each computers. Then, computer network speed level would be determined and stored in server. Server would split the whole system into many subsystems and define $Q$ particles;

(2) Parameters $x, y, \theta$ would be set with random numbers, and splitting line should be constructed. The whole education system would be divided into $Q \times 2$ subsystems. For the reason of location of nodes in the network is known, $c_{i}(i=1,2)$ of each node can be determined and used to calculate the value of fitness .

(3) Value of fitness would be compared with minimum fitness value which is in the last round, and the less one is elected to be the general extreme value of ${P_{g d}}_{g d}$; al the fitness value of individual node would be taken into comparison, and the least value is elected to be individual extreme value of $P_{i d}$, then $x, y, \theta$ should be apdated:

Where,

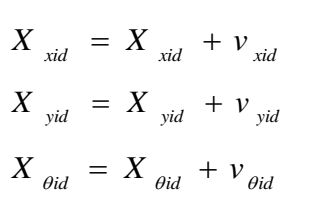

$X_{x i d}, X_{y i d}$ represents the particle location;

$X_{\text {oid }}$ represents the angular of the splitter;

$v_{x i d}, v_{\text {yid }}, v_{\theta i d}$ represents the search speed in three dimensions.

And all the parameters above can be determined by following equations:

$$
\begin{aligned}
& v_{x i d}=\omega v_{x i d}+c_{1} \times \text { rand } O\left(P_{\text {tid }} \backslash X_{x i d}\right)+c_{2} \times \text { rand }\left(O\left(P_{g d}-X_{x i d}\right)\right. \\
& v_{y i d}=\omega C_{y i d}+c_{1} \times \text { rand }\left(O P_{2}-X_{y i d}\right)+c_{2} \times \text { rand } O\left(P_{g d}-X_{y i d}\right) \\
& v_{\theta i d}=\omega v_{\theta i d}+c_{1} \times \operatorname{rand} \theta\left(P_{i d}-X_{\theta i d}\right)+c_{2} \times \operatorname{rand}()\left(P_{g d}-X_{\theta i d}\right)
\end{aligned}
$$

Where, $c_{1}$ and $c_{2}$ represent the study factor, and $c_{1}=c_{2}=2$; rand () means random number between 0 and 1 ; $\omega$ means the weighted factor.

(4) After the update of parameters $x, y, \theta$, jump to step 2 to continue the research process. When the fitness value is 0 or maximum search times have been approximate, process would be mandatory finished. In ideal conditions, When the fitness value is approximately 0 , the whole system would be divided into two subsystems.

(5) Use the same method to divide the subsystem, until get proper subsystem number.

\section{Step 3: Search the fastest multi-hop path by ACO}

Some definitions must be given firstly.

Definition 1: $R\left(v_{i}\right)$ represents area covered with $\operatorname{computer}_{v_{i}}$. Forward area represents computers with higher network speed than the computer $v_{i}$ itself.

Definition 2: Forward neighbor computer are computers in the forward area of $\operatorname{computer}_{V_{i}}$, and those computers all belong to $\operatorname{set}_{N_{f}}\left(v_{i}\right)$. 
In this step, the first work is to determine the center computer. We define computer with the highest network speed as the center computer. Other computers with relatively fast network speed are vice center computers.

This search is based on single subsystem, so the path in each subsystem has some difference, and one subsystem may have more than one search paths. One search path would have no effect on others. That means that path search is independent in each subsystem and path search is independent even in same subsystem. Each computer would send data to center computer and vice center computers to ensure location.

According to the definition above, forward neighbor nodes can be marked as the following:

$$
N_{f}(i)=\left\{v_{j} \mid v_{j} \in V, d_{i, j} \leq r, \alpha_{i, j} \leq \beta_{i}\right\}
$$

Each computer would separate a place in the disk to store location and network speed information and separate a place in memory to store the information between self and neighbor computers, including self-network speed, neighbor computer network speed, and so on. Aiming at representing pheromone, each computer woyld take a parameter $\tau_{i j}$ to record the pheromone density. Ant in the education sys em s'a data package with capability of memory and storage. The ant should have characteristies listed in the following:

(1) The ant can store the information of computers it passed;

(2) The ant should store the computers itpassed with a proper sequence and forming a path. When the ant passes or returns, it wouldupdate the phoromone.

(3) Ant just can jump to neighbor nodes with higher network speed;

(4) Ant has the capability of reading and modifying information it locates.

Ants in the education system would beginat computers which want to fetch data from server and go to the center computer withroulti-hop mechanism to find nearest path between computer and center computers or Kice/center computers. Ant locates at $V_{i}$ should determine the jump probability $P_{i}{ }^{k}$ with information of pheromone and network speed of the neighbor computers. $P_{i, j}^{\prime}$ represents the probability of ant $k$ jump to forward neighbor computer.

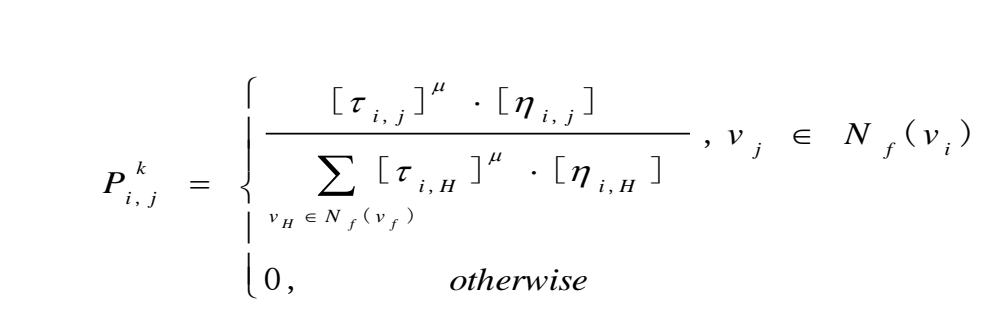

Where, $\tau_{j}$ is the network speed value of path $l_{i, j}$ from node $v_{i}$ to its neighbor node $_{V_{j}}$; $\mu$ is a factor influenced by network speed and pheromone. It means the influence of information accumulated on the path of ants. The bigger the value of $\mu$ is, the faster path the ant would choose. At first time $t_{0}$, pheromone $\tau_{i, j}\left(t_{0}\right)$ on the path ${ }_{i_{i, j}}$ of each computer. It can be calculated as:

$$
\tau_{i, j}\left(t_{0}\right)=\frac{d_{i, D}}{d_{i, j}+d_{j, D}} \times\left(1-\frac{d_{i, j}}{\sum_{v_{H} \in N_{f}\left(v_{i}\right)} d_{i, H}}\right)
$$

$\eta_{i, j}$ is a function of data processing speed, which can be calculated as following: 


$$
\eta_{i, j}=\frac{e_{j}}{\sum_{v_{H} \in N_{f}\left(v_{i}\right)} e_{H}}
$$

Where, $e_{H}$ is data processing speed of forward neighbor node $v_{H} \in N_{f}(H)$ of node $i$. Forward neighbor computers set limit the jumped objection. After the $v_{j}$ has been elected as the next computer of $V_{i}$ jumped, it would be calculated according to the network speed of $v_{j}$. Following the same method, the ant would jump to the center computer or vice center computers. Due to the mechanism of subsystem dividing, one computer in a subsystem would have forward neighbor computer. And center computer won't have forward neighbor computer. It would contact with proxy or server directly. At the same definition, computers, which have no forward computer, would directly contact with center computer or vice computers. If one computer finds a short path from the neighbor subsystem, it can send data through neighbor subsystem when the neighbor subsystem is not/in a busy state. An ant travels from source computer to center computer and return back to the source computer with the pheromone information updated is defined to be a whole process. All ants finish a process, one loop is completed and value of loop index thes 1 . Pheromone density in the path $i_{i j}$ would be adjusted timely in the loop $t$.

In the equation, $m$ represents the ants number; ${ }_{\left.\Delta \tau_{i, j}{ }^{k}\right)}$ means pheromone left on path $l_{i j}$ at loop $t ; L^{k}{ }^{k}(t)$ is the lengfh of ant $k$ moves at loop $t ; Q$ is a constant about pheromone. $\rho$ is also a constant which describe the volatilization of pheromone. Value of $\rho$ is often between 0 and 1 to keep the convergence of the algorithm.

\section{Simulationand Verification}

In an edueation system, there have one server and three proxy server. There are 32 thousand computers in the system, while 200 computers are in the highest speed level, 1239 computers are in middle speed level and the rest in the lowest speed level. The total bandwidth is $1000 \mathrm{M}$. We simulate 12.5 thousand computers are online at the same time. And the total system is divided into 76 subsystems. Each subsystem can get different bandwidth. So, we select one computer randomly to study the results of the new algorithm.

In the algorithm, the computer can use other high network speed computer to fetch data segment and pre-store them, and get the data to keep the video or file transfer fluency. 


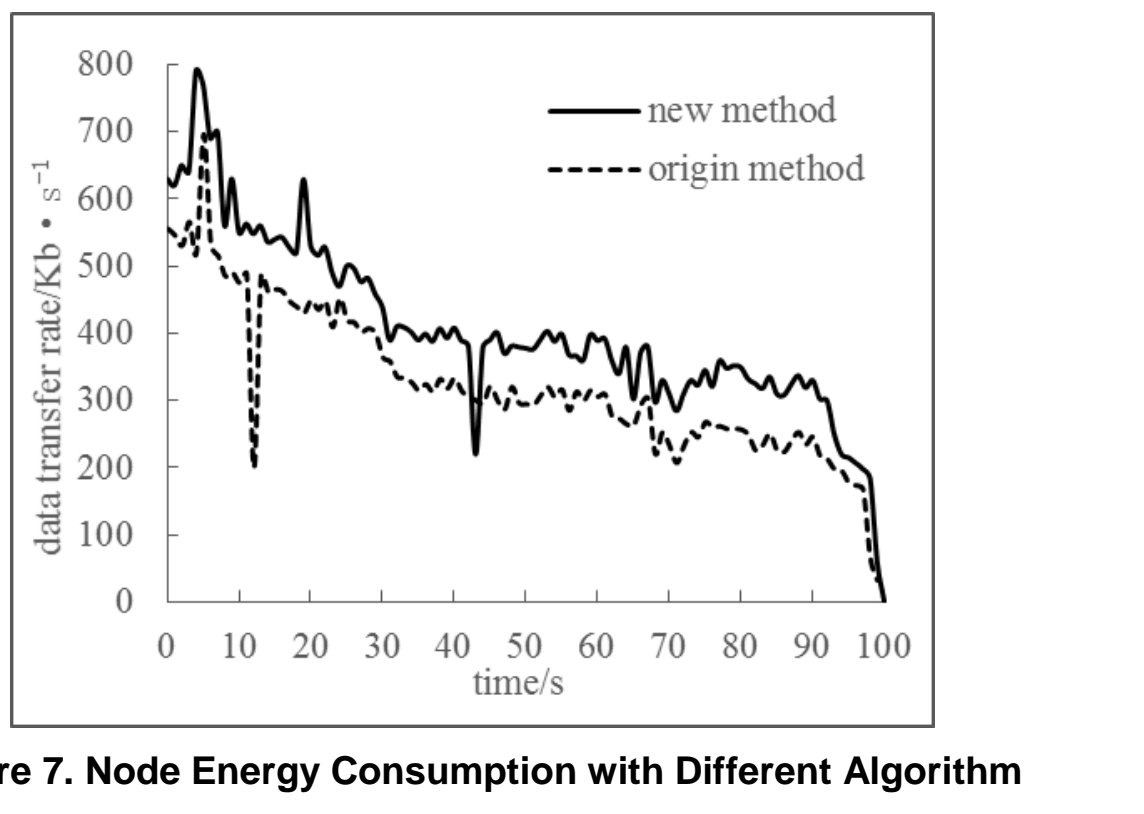

From the Figure 7, we can see that the data transfer rate has been greatly improved. With the new data transfer method, we can play educationabvideo much more fluency and reduce the delay time. The simulation result shows that the new method is effective.

\section{Conclusion}

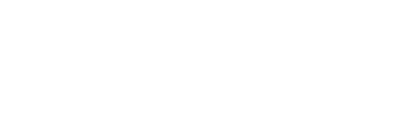

The paper proposes a new method to improve the data transfer speed in education network system. The method is based on multi hop mechanism, but it is different from multi hop mechanism in WSN. The method firstly uses PSO to divide the whole education system into more than one subsystems, and then ACO is used to find the fastest path to transfer the data package. In the path searching period, the ant would go to center computer and vice computers along paths with the)fastest speed and the path would be more than one to improve the data transfer speed. Results of the simulation show that, the new algorithm is an effective method for data transferon an equation system with different network speed area. It can be firstly used in the education system and extend the application to other fields.

\section{References}

[1] L. M. Brenda, A. L. Degennaro, "Computer graphics for art creation: Cultural biases against its acceptance in education", Computers and Graphics (Pergamon), vol. 23, no. 3, (1999), pp. 419-427.

[2] W. Chaoshun, "Effects of net-media on art design education", 2nd International Workshop on Education Technology and Computer Science, ETCS 2010, vol. 2, (2010), pp. 719-722.

[3] N. Diep, M. Jolie, L. Valerie, P. Jonathan and K. Michelle, "Shrink-film micro fluidic education modules: Complete devices within minutes. Biomicrofluidics, vol. 5, no. 2, (2011).

[4] M. F. Iskander, "Multimedia- and computer-based engineering education", Annual Review of Progress in Applied Computational Electromagnetics, vol. 1, (1999), pp. 212-216.

[5] K. Hiroyuki and I. Shintaro, "Tool based multimedia distance learning system for engineering education", Proceedings of the IEEE International Conference on Systems, Man and Cybernetics, vol. 2, pp. 239-242. (1999).

[6] A. I. Reuther, D. G. Meyer, "The effect of personality type on the usage of a multimedia engineering education system", Proceedings - Frontiers in Education Conference, vol. 1, (2002), pp. T3A/7-T3A/12.

[7] K. J. Jaromír, K. Zdravko, V. P. Sabev, "Lam Hon Loong", Advanced multimedia engineering education in energy, process integration and optimization. Applied Energy, vol. 101, (2013), pp. 33-40. 
[8] Z. Z. Hanim, W. Ahmad and W. Fatimah, "A study on students' motivation in learning mathematics using multimedia courseware", Proceedings 2010 International Symposium on Information Technology - Visual Informatics, ITSim', vol. 10, no. 1, (2010).

[9] Z. Jing, "Multimedia technology in mathematics teaching optimization", Advances in Intelligent and Soft Computing, 117 AISC 127, vol. 2, (2012), pp. 357-363.

[10] J. Xu, N. Jin, X. Lou, T. Peng, Q. Zhou and Y. Chen, "Improvement of LEACH protocol for WSN", 2012 9th International Conference on Fuzzy Systems and Knowledge Discovery (FSKD 2012), (2012), pp. 2174-2177.

[11] W. Wei, P. Shen, L. Zhang, H. Xu, J. Song, W. Zhang and W. Wang, "LEACH-Based Energy-Conserved Improved Protocol for WSNs", International Journal of Digital Content Technology and its Applications (JDCTA), vol. 6, (2012), pp. 163-171.

[12] W. Luan, C. Zhu, B. Su, C. Pei, "An Improved Routing Algorithm on LEACH by Combining Node Degree and Residual Energy for WSNs", IOT Workshop 2012, CCIS, vol. 312, (2012), pp. 104-109.

[13] B. A. Bakr and L. Lilien, "A Quantitative Comparison of Energy Consumption and WSN Lifetine for LEACH and LEACH-SM", 2011 31st International Conference on Distributed Computing Systems Workshops, (2011), pp. 182-191.

[14] P. Xue-feng and L. La-yuan, "Design of an Energy Balanced Based Routing Protocol for WSN", 2011 IEEE, (2011), pp. 366-369.

[15] R. K. Kodali and N. Sarma, "NVS. Energy Efficient Routing Protocols for WS ('s"), 2013 International Conference on Computer Communication and Informatics (ICCCI 2013), (2013).

[16] A. Ahlawat, V. Malik, "An EXTENED VICE-CLUSTER SELECTION APPRØACH TO IMPROVE V LEACH PROTOCOL IN WSN", 2012 Third International Conference on Advanced Computing \& Communication Technologies, (2013), pp. 236-240.

[17] Xu Long-long, Zhang Jian-jun. Improved LEACH Cluster Head Mutti-hops Algorithm in Wireless Sensor Networks. Ninth International Symposium eon Distríbuted Computing and Applications to Business, Engineering and Science, 10-12. (2010)

[18] N. Jan, K. Ryszard, N. MacIej and C. Zenon "Multi-hop and directed routing based on neighborhood cooperation in WSN", INES 2011 - 15th International Conference on Intelligent Engineering Systems, (2011), pp. 221-227.

[19] Y. Shi and R. C. Eberhart, "A modified particla swarm optimizer", Proceedings of IEEE International Conference on Evolutionary Computation, (1998), pp.69-73.

[20] I. Vilovic, N. Burum, Z. Špus and R. Nad, "PSO and ACO algorithms applied to location optimization of the WLAN base station", ICECOm 2007, 19th International Conference on Applied Electromagnetics and Communications, (2007), ppy $1-5$.

[21] K. M. Sim and W.H. Sur, "Ant Colony Optimization for Routing and Load-Balancing: Survey and New Directions", IEEE Transactions on Systems, Man, and Cybernetics, Part A, vol. 33, no. 5, (2003), pp. 560572.

[22] S. D. Dissanayake and J. Arnstrong, "Novel techniques for combating DC offset in diversity combined ACO-OFDM", IEEE Communications Letters, vol. 5, no. 11, (2011), pp. 1237-1239.

[23] Sherafat, Elham, Hafeż, Leila, Shekarian and M. Hossein, "A Modified ACO: Improving performance by avoiding premature convergence", Proceedings of the 2008 International Conference on Genetic and Evolutionary Methods, GEM 2008, (2008), pp. 158-161.

[24] J. Pan, L. Cai, Y. T.Hou, Y. Shi and S. X. Shen, "Optimal base station locations in two-tiered wireless sensor networks", IFEE Transactions on Mobile Computing, vol. 4, no. 5, (2005), pp. 458-473.

[25] G. Wang G. Cáo, T. La Porta and W. Zhang, "Sensor relocation in mobile sensor networks", In Proceedings of the 24 th International Annual Joint Conference of the IEEE Computer and Communications Societies (INFOCOM05) Miami, FL. (2005).

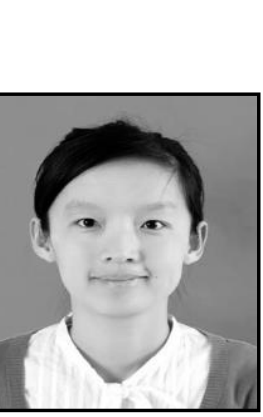

\section{Authors}

Zhe Zhou, she received her bachelor's degree of science in Langfang Teachers University, Langfang, Hebei in 2005 and master's degree of Educational Technology in Hebei University (2009). Now she is a lecturer in Hebei Normal University of Science \& Technology, Qinhuangdao, Hebei. Her major fields of study are instructional design, media teaching theory and distance education. 


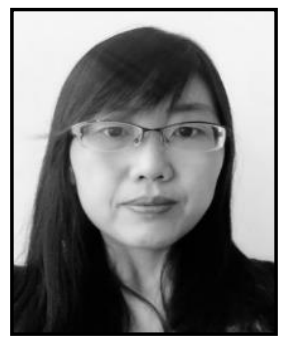

Jianying Li, she received her bachelor's degree of education in Hebei Normal University, Shijiazhuang, Hebei in 2005 and master's degree of computer technology in Yanshan University, Qinhuangdao, Hebei. (2011). Now she is a lecturer in Hebei Normal University of Science \& Technology, Qinhuangdao, Hebei. Her current research interests include education technology and vocational education.

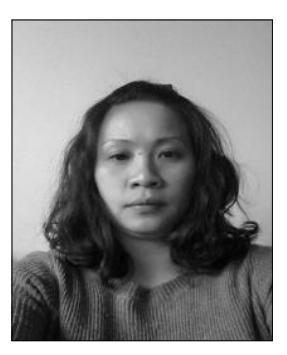

Shuang Chen, she received her bachelor's degree of engineering in Tianjin University of Technology and Education, Tianjin (2003) and master's degree of engineering in Guizhou University (2007), She is studying for a PhD in Philosophy of science and echnology at the Huazhong University of Science and technology. Nowshe is a lecturer in Hebei Normal University of Science \& Technoløgy, Qinhuangdao, Hebei. Her major fields of study are educational technology and innovation education.

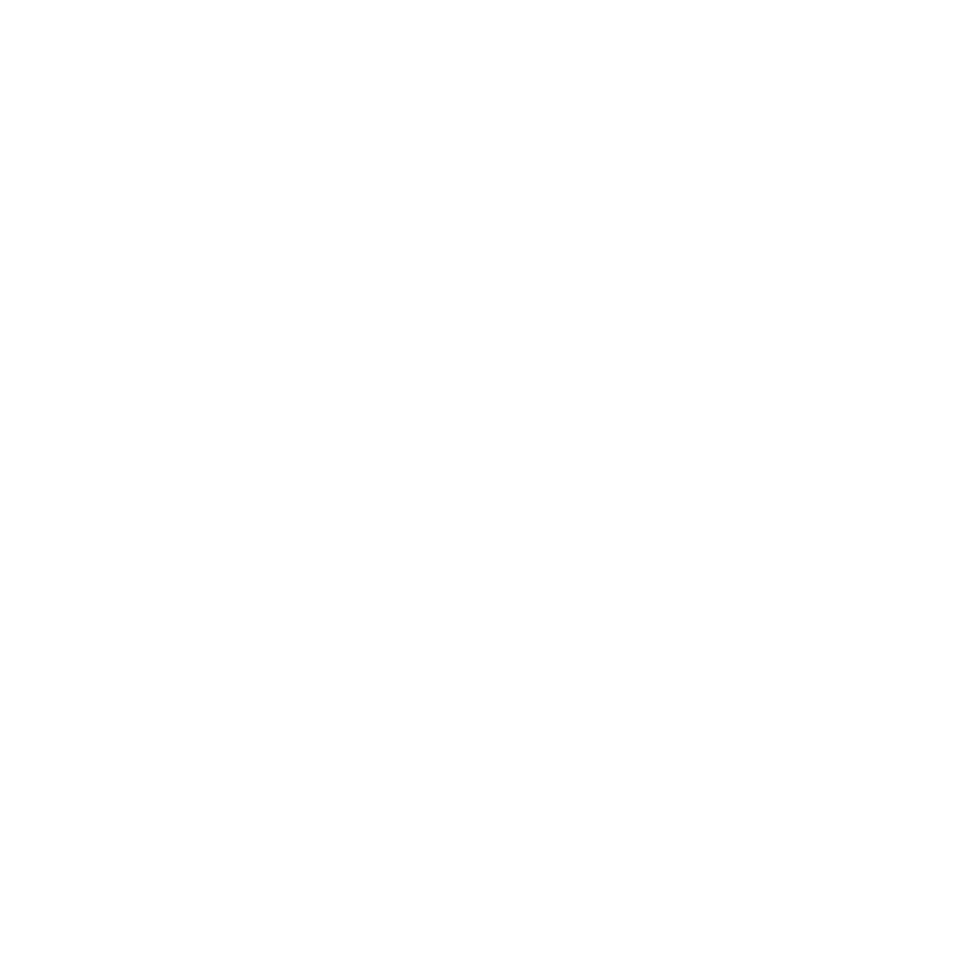


International Journal of Multimedia and Ubiquitous Engineering Vol.9, No.5 (2014)

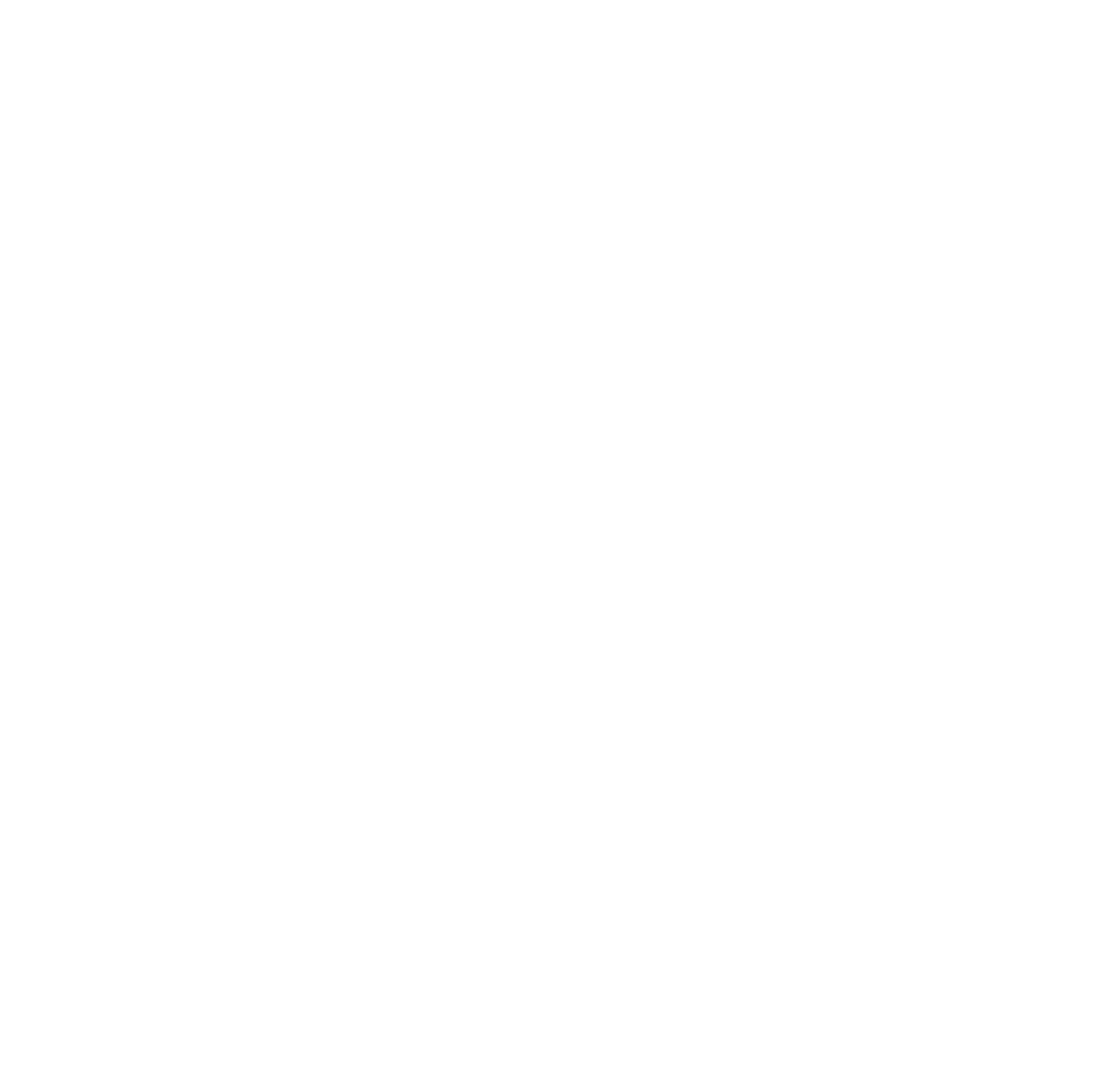

